\title{
A Case of a Temporary Endotracheal Stent for Airway Management in a Patient with Primary Thyroid Lymphoma
}

\author{
Young Chul Kim, Seong-Chul Yeo, Jin Pyeong Kim, and Jung Je Park \\ Department of Otorhinolaryngology-Head and Neck Surgery, Institute of Health Sciences, Gyeongsang National University \\ School of Medicine, Gyeongsang National University Hospital, Jinju, Korea
}

\section{원발성 갑상선 림프종 환자의 기도확보를 위한 Endotracheal Stent 삽입 증례}

김영철· 여성철· 김진평· 박정제

경상대학교 의과대학 경상대학교병원 이비인후과학교실, 건강과학연구원

\author{
Received April 25, 2019 \\ Revised June 2, 2019 \\ Accepted June 17, 2019 \\ Address for correspondence \\ Jung Je Park, MD, PhD \\ Department of Otorhinolaryngology- \\ Head and Neck Surgery, \\ Institute of Health Sciences, \\ Gyeongsang National University \\ School of Medicine, Gyeongsang \\ National University Hospital, \\ 501 Jinju-daero, Jinju 52828, Korea \\ Tel $+82-55-750-8698$ \\ Fax +82-55-759-0613 \\ E-mail capetown@hanmail.net
}

Primary thyroid lymphoma is a rare malignancy that can grow rapidly and compress the airway. In such cases, a tracheostomy or thyroidectomy is generally required. Here, we report a case of a 76-year-old woman diagnosed with primary thyroid diffuse large B-cell lymphoma (DLBL), with dyspnea attributed to tracheal compression by the enlarged thyroid. The patient's poor general condition meant that thyroidectomy was considered challenging. Therefore, a temporary endotracheal silicone stent was successfully inserted to maintain the airway, as tracheal stenting has been shown to be an effective treatment for both benign and malignant obstructive airway diseases. We conclude that, in patients with primary thyroid DLBL who present with airway compression but are not eligible for surgery, tracheal stent insertion can maintain the airway and allow time for chemotherapy to be administered.

Korean J Otorhinolaryngol-Head Neck Surg 2020;63(2):85-90

Key Words Airway management · Diffuse large cell lymphoma · Palliative care · Thyroid cancer.

\section{Introduction}

Primary thyroid lymphoma is a rare disease that accounts for less than $5 \%$ of all thyroid malignancies. ${ }^{1)}$ According to the current classification system, diffuse large B-cell lymphoma (DLBL) is the most common subtype. ${ }^{2)}$ One of the important features of thyroid lymphoma is that the tumor can grow rapidly over the course of only a few months, leading to airway obstruction. ${ }^{3)}$ In such cases, an emergency tracheostomy or early thyroidectomy is usually performed to secure the airway. Airway stenting is a type of interventional bronchos-

This is an Open Access article distributed under the terms of the Creative Commons Attribution Non-Commercial License (https://creativecommons.org/licenses/by-nc/4.0) which permits unrestricted non-commercial use, distribution, and reproduction in any medium, provided the original work is properly cited. copy that is widely used for the treatment of benign and malignant airway problems. Currently, a wide range of stent types (such as metal, silicone, self-expanding metal, and hybrid stents) are available for the management of different clinical conditions and their application has been shown to be effective in the treatment of malignant lesions, despite some side effects such as stent migration or malposition, granulation formation, infection, mucoid impaction. ${ }^{4}$

Here, we report the recent case of a patient with primary thyroid DLBL who presented with airway compression but was not considered eligible for surgery. Therefore, a temporary tracheal silicone stent was inserted to allow time for chemotherapy to be administered. Along with a review of the literature, we report on the potential value of this ap- 
proach in patients undergoing chemotherapy for primary thyroid DLBL.

\section{Case}

A 76-year-old woman presented with a 5 day history of dyspnea and was admitted to the pulmonology department of the Gyeongsang National University Hospital. Chest CT scanning showed an enlarged thyroid gland and narrowing of the upper airway, and the patient was referred to the otorhinolaryngology department. While taking the patient's history, it was noted that she had been diagnosed with Hashimoto's thyroiditis 6 years previously and had been undergoing regular follow-up visits at the endocrinology department. An anterior neck mass had rapidly increased in size within the last 3 months. A diffusely enlarged thyroid gland was identified, but there were no palpable lymph nodes. Normal vocal cord movement was observed, and rigid laryngoscopic examination showed no lesions that may have accounted for the dyspnea (Fig. 1A).

An additional bronchoscopy was performed to evaluate the upper airway, which showed that the endotracheal lumen was reduced by $70-80 \%$ due to external compression and localized ulcerating mucosal infiltration (Fig. 1B). During biopsy of the mucosal lesion, tracheal spasm resulted in hypoxia and subsequent cardiac arrest, requiring cardiopulmonary resuscitation to restore the patient's spontaneous circulation. To obtain a more
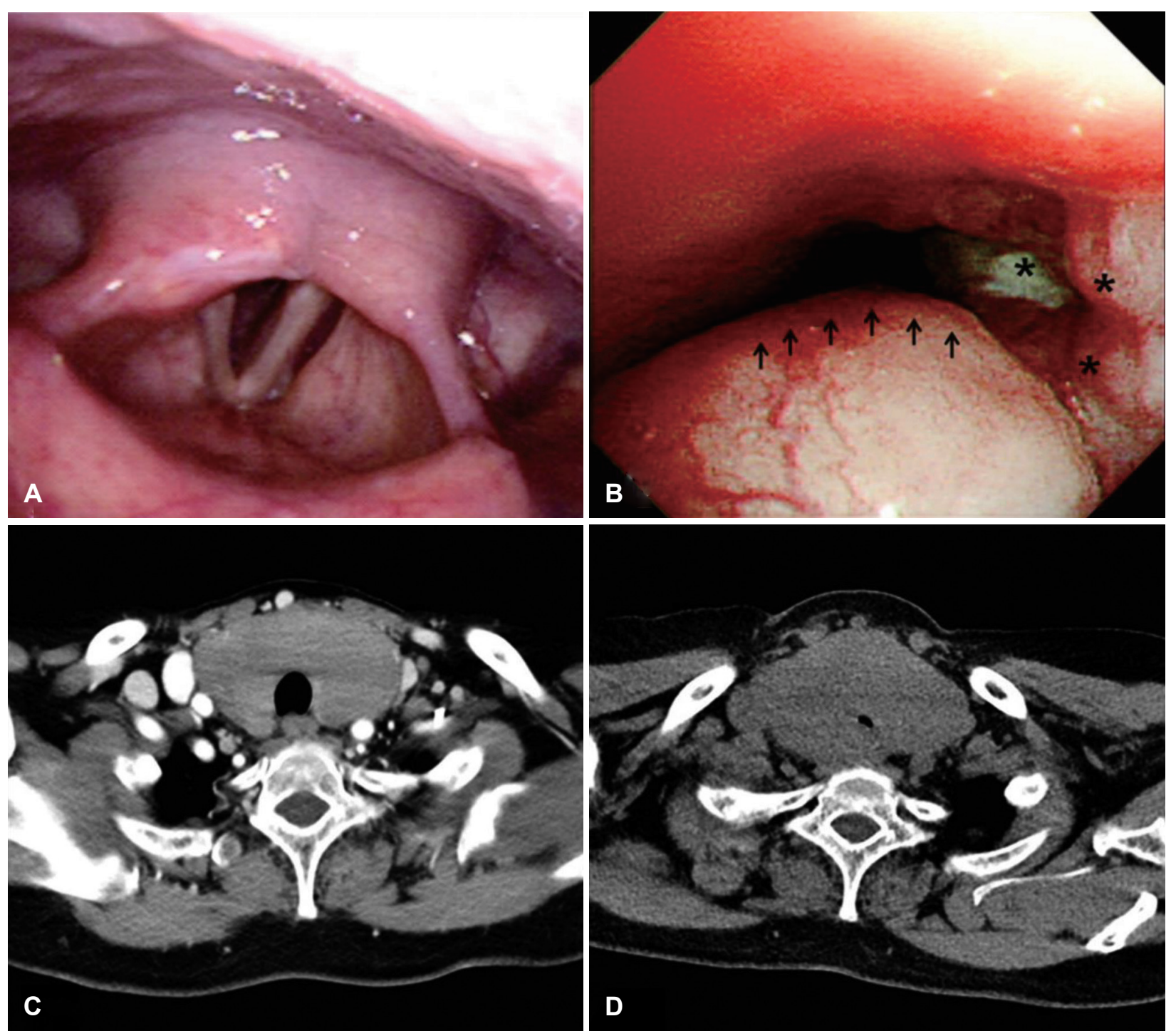

Fig. 1. Endoscopic findings and CT scan images of patient. Rigid laryngoscopic examination showing a normal larynx (A). Bronchoscopic findings showing severely narrowed tracheal lumen owing to the presence of a mass (arrows), and irregular mucosa with partial ulcerative lesion (asterisks)(B). Chest CT scan image obtained 3 months before the onset of symptoms (C). Pharynx CT scan image showing enlarged thyroid gland and severely narrowed tracheal lumen (D). 

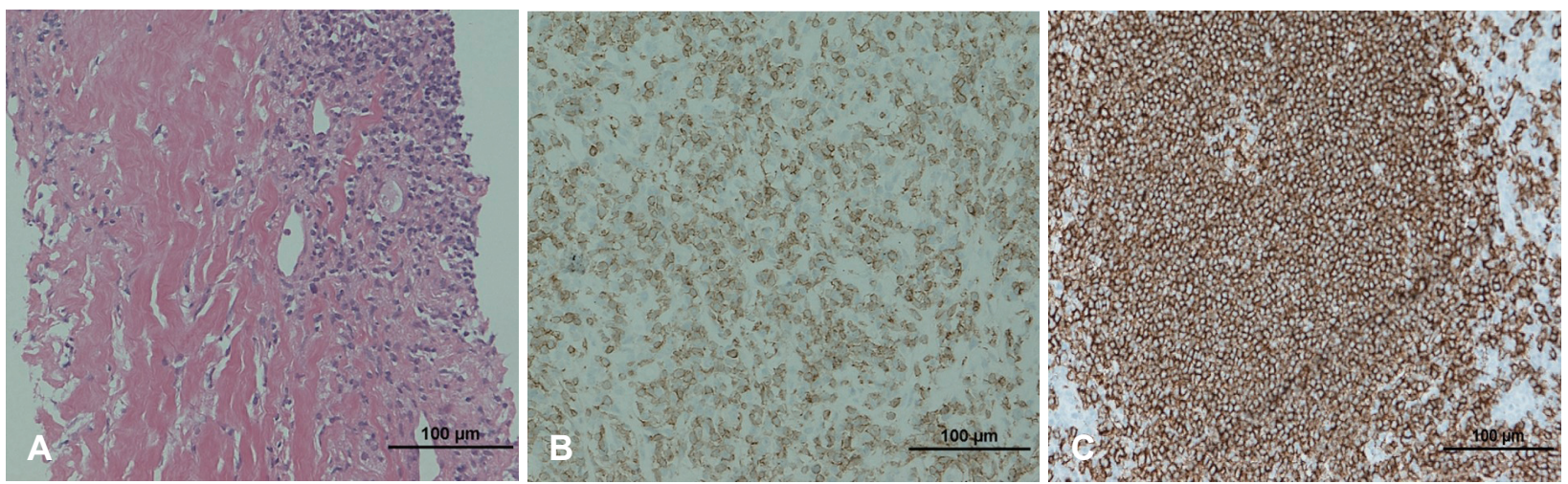

Fig. 2. Microscopic findings of the thyroid mass showing diffuse infiltration of the lymphoid cells (hematoxylin and eosin staining, $\times 200$ ) (A). Weak CD3 positivity (B), strong CD20 positivity (C)(B and C: immunohistochemical staining, $\times 200)$.

accurate radiological diagnosis, contrast-enhanced CT of the pharynx was performed, showing a notable increase in the size of the thyroid gland, tracheal deviation, and luminal narrowing when compared with chest CT scans obtained approximately 3 months earlier (Fig. 1C and D). A histological diagnosis was established by ultrasound-guided core needle biopsy, performed while the patient was intubated for airway maintenance. Hematoxylin and eosin staining showed diffusely infiltrated atypical lymphoid cells, which were shown to be strongly CD20-positive and weakly CD3-positive by immunohistochemical staining (Fig. 2). On the basis of these results, the patient was diagnosed with primary thyroid DLBL. Additional radiographic studies were performed to determine the stage of the disease, which showed involvement of the upper esophagus, bilateral cervical II, III, and IV lymph nodes, and bilateral supraclavicular lymph nodes, as well as the thyroid gland.

While surgery was considered to de-bulk the thyroid gland and secure the airway, the risks associated with a general anesthesia were deemed to be too high, considering the patient's age, difficulty with intubation, and underlying diseases (such as hypertension, diabetes, asthma, atrial fibrillation, and history of cerebral infarction). Following a multidisciplinary team meeting, airway maintenance with endotracheal intubation during chemotherapy was agreed to be the preferred approach; however, the patient and her family insisted on stenting and an endotracheal silicone stent insertion was performed by a pulmonologist. Rigid bronchoscopy was performed under general anesthesia. After mechanical widening with serial rigid tubes, stent was placed in the stenotic trachea. Stent was a diameter of $12 \mathrm{~mm}$ and length of $50 \mathrm{~mm}$ considering normal diameter and narrowed vertical length of trachea.

The patient's dyspnea resolved immediately after stent insertion, and chemotherapy with the rituximab, cyclophospha- mide, hydroxydaunorubicin, vincristine, prednisolone (RCHOP) regimen was initiated after 6 days. The plan was to remove the stent as soon as possible to avoid any associated complications; no events were noted up to 4 weeks after the start of chemotherapy. However, at 5 weeks, the patient complained of dyspnea, and pharyngeal CT was performed to identify the cause. This showed a significant decrease in the size of the thyroid gland and an absence of tracheal deviation and narrowing, but a soft tissue shadow was noted inside the stent and further exploration under suspension laryngoscopy was performed immediately (Fig. 3A). The narrowing of the stent lumen was found to be due to a sticky mucous plug, which was removed by repeated suction; no other findings were noted (Fig. 3B and C). The absence of tracheal compression was noted on both radiological and laryngoscopic investigation, and the stent was removed by a pulmonologist and the endotracheal lumen was widened enough to secure the airway (Fig. 3D).

While the patient's dyspnea resolved after the removal of the stent, the patient developed neutropenia during preparation for the third cycle of chemotherapy and chemotherapy was discontinued. Subsequently, the patient developed pneumonia due to carbapenem-resistant Klebsiella pneumoniae infection. The patient's general condition followed a repeated pattern of improvement and deterioration and she eventually died.

\section{Discussion}

Primary thyroid lymphoma is a rare disease that accounts for less than $5 \%$ of all thyroid malignancies and $1-2.5 \%$ of all lymphomas. ${ }^{1)}$ Under the revised European-American classification of lymphoid neoplasm and World Health Organization classification systems, it is now classified into 

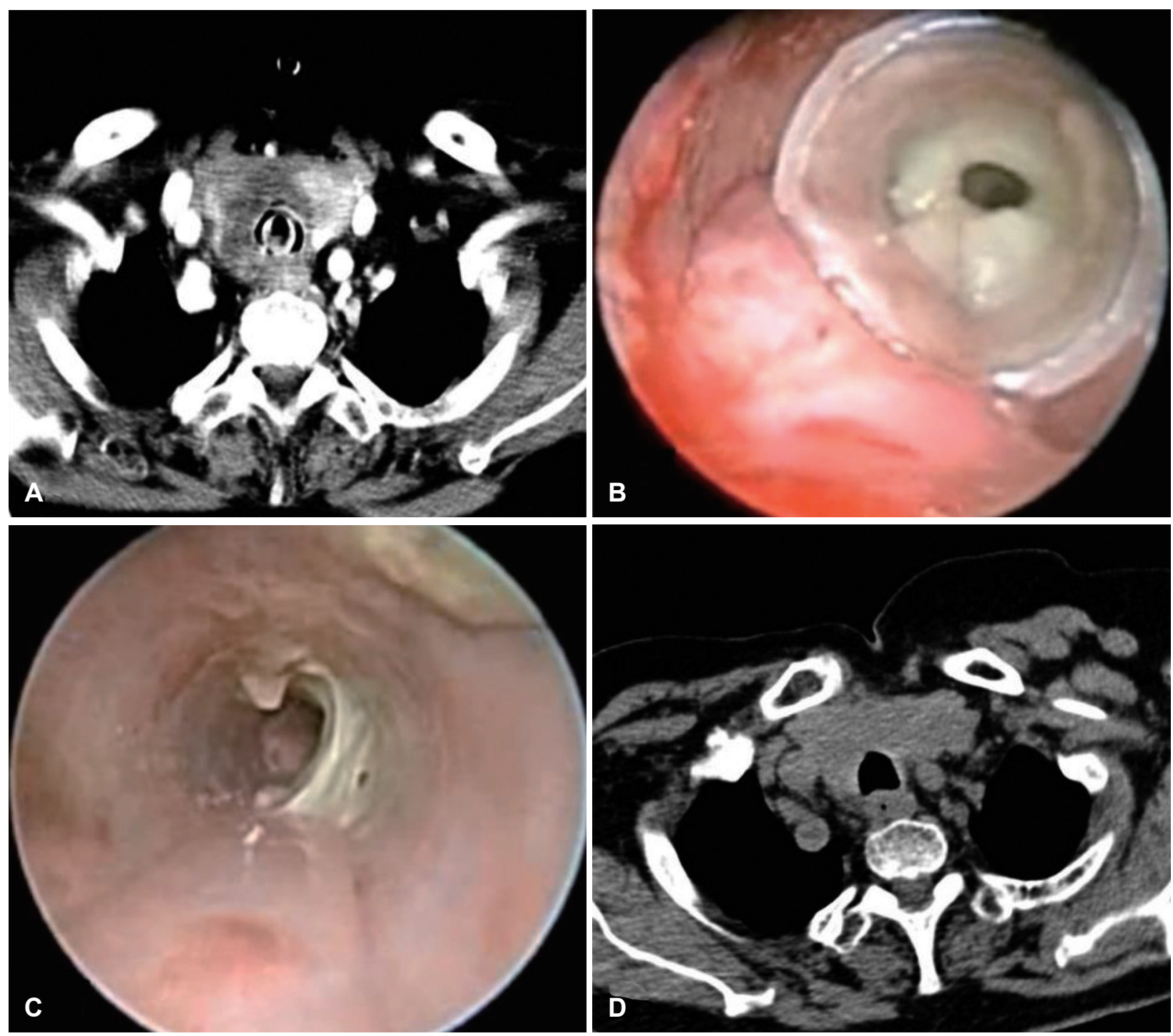

Fig. 3. Endoscopic findings and CT scan images after endotracheal stent implantation. An enhanced pharynx CT image showing a widened tracheal lumen secondary to the decreased thyroid gland and endo-stent soft tissue lesion (A). Endoscopic view showing stent narrowing due to mucous plugging (B). Endoscopic view after the mucous plug had been removed (C). An enhanced pharynx CT image showing a widened tracheal lumen after endotracheal stent was removed (D).

DLBL, extra-nodal marginal zone B-cell lymphoma, and other rare subtypes. ${ }^{5}$ Of these subtypes, DLBL is the most common variant. ${ }^{2)}$ As the thyroid gland lacks lymphoid tissues, most thyroid lymphomas arise from pre-existing chronic autoimmune thyroiditis. ${ }^{6}$ The incidence of DLBL is approximately 67 times higher in patients with Hashimoto's thyroiditis. ${ }^{7)}$

Unlike most thyroid cancers, which are generally well-differentiated and slow-growing, anaplastic cancer and malignant lymphomas can grow rapidly and cause tracheal compression; emergent tracheostomy or early thyroidectomy may therefore be required to secure the airway. ${ }^{3,8)}$ The case presented here also showed rapid deterioration within 2-3 months. In terms of diagnosis, fine needle aspiration is often used to evaluate the thyroid mass, although the diagnostic accuracy is only approximately $60 \%$ due to histological similarities to Hashimoto's thyroiditis. ${ }^{9)}$ Therefore, in such cases, additional core needle biopsy or thyroidectomy may be required.

The treatment of primary thyroid lymphoma varies according to the subtype, histological findings, size, stage, and comorbidities. For DLBL, three cycles of R-CHOP is considered to be standard treatment; involved-field radiation therapy may be included in patients with advanced disease. $^{10,11)}$ The current role of surgical management is primarily for the purpose of histological diagnosis and securing the airway, with a treatment goal being appropriate in only a lim- 
ited number of cases. In the case presented here, surgical management was considered both for histological confirmation and to secure the airway. However, due to the patient's age, pre-existing comorbidities, and severe tracheal narrowing, general anesthesia was considered to pose a high risk. In addition, the patient and her family wanted to avoid surgery if possible as the patient had experienced cardiac arrest during bronchoscopy. Following discussion by a multidisciplinary team, the decision was taken to proceed with chemotherapy under endotracheal intubation, although due to the patient's preference for endotracheal stenting, this procedure was subsequently performed.

Tracheal stenting techniques have progressed in recent years, and a variety of stents are under development. ${ }^{4)}$ To date, few reports of stent insertion in patients with thyroid disease have appeared in the literature. In 2013, Shin, et al. ${ }^{12)}$ reported a successful case using a self-expandable metal stent and radiofrequency ablation therapy for a patient with multinodular thyroid goiter; Ding, et al. ${ }^{13)}$ reported excellent results using self-expanding metal stents for the treatment of mucosa-associated lymphoid tissue lymphoma. Madan, et al. ${ }^{14)}$ and Varadharajan, et al. ${ }^{8)}$ have also reported the usefulness of tracheal stenting in patients with anaplastic thyroid carcinoma. By contrast, Cho, et al. ${ }^{15)}$ noted serious complications after stent insertion in a patient with malignant lymphoma, and suggested that self-expandable metal stents should not be used in benign airway disease, with silicone stents being preferable in patients with benign or curable malignant airway disease.

Metal stents are usually net-shaped and are folded when inserted. They are self-expanding and resistant to external pressure, and show minimal displacement. However, the tumor can grow into the lumen of the stent and, therefore, removal can become difficult. By contrast, silicone stents are easy to adjust and remove, but are susceptible to external pressure and are easily displaced; therefore, various adverse events, such as stent displacement, granuloma formation, mucous plugging, bacterial growth, tumor ingrowth, and failure of adaptation, are known to occur. ${ }^{4)}$ In the current case, we intended to reduce the risk of complications by maintaining the stent for the shortest time possible. A silicone stent was selected due to concerns regarding tumor ingrowth and the fact that these stents are easy to handle and remove.

In the case presented here, chemotherapy was initiated 6 days after stent insertion and no complications were observed until two cycles of chemotherapy had been completed. How- ever, the patient complained of dyspnea approximately 5 weeks after stent insertion. CT evaluation revealed a mucous plug in the lumen of the stent but also confirmed a remarkable decrease in the size of the thyroid lymphoma; the stent was subsequently removed successfully. Although there is the possibility that mucous stasis and bacterial colonization in the endotracheal stent contributed to the development of pneumonia, we consider that neutropenia as a complication of chemotherapy is likely to be the most probable cause. For such complications, Cho, et al. ${ }^{15)}$ have recommended surveillance examination with bronchoscopy 4-6 weeks after tracheal stent insertion regardless of symptoms and reported that the incidence of adverse events can be significantly reduced using this approach. Although our patient died as a result of developing pneumonia, this case has demonstrated the feasibility of carrying out chemotherapy after securing the airway through temporary stent insertion in patients with primary thyroid DLBL who are not eligible for surgery.

In patients with thyroid DLBL who present with airway compression but are not eligible for surgery, temporary endotracheal stent insertion can be an effective alternative that can secure the airway and enable continuous chemotherapy. In such cases, a stent can be maintained more safely through surveillance via bronchoscopy.

\section{Author Contribution}

Conceptualization: Jung Je Park. Data curation: Young Chul Kim, Seong-Chul Yeo, Jin Pyeong Kim, Jung Je Park. Investigation: Young Chul Kim, Seong-Chul Yeo. Methodology: Young Chul Kim, SeongChul Yeo, Jin Pyeong Kim, Jung Je Park. Supervision: Jung Je Park, Jin Pyeong Kim. Validation: Young Chul Kim, Seong-Chul Yeo, Jin Pyeong Kim, Jung Je Park. Writing — original draft: Young Chul Kim, Seong-Chul Yeo, Jung Je Park. Writing—review \& editing: Young Chul Kim, Seong-Chul Yeo, Jung Je Park.

\section{ORCID}

Jung Je Park

https://orcid.org/0000-0001-6325-0398

\section{REFERENCES}

1) Ha CS, Shadle KM, Medeiros LJ, Wilder RB, Hess MA, Cabanillas $\mathrm{F}$, et al. Localized non-Hodgkin lymphoma involving the thyroid gland. Cancer 2001;91(4):629-35.

2) Alyami H, Alsofyani T, Bu Bshait M, Al-Osail EM. Primary Diffuse B-Cell Thyroid Lymphoma: Case Report and Literature Review. Case Rep Oncol 2018;11(2):505-10.

3) Shen H, Wei Z, Zhou D, Zhang Y, Han X, Wang W, et al. Primary extra-nodal diffuse large B-cell lymphoma: A prognostic analysis of 141 patients. Oncol Lett 2018;16(2):1602-14.

4) Folch E, Keyes C. Airway stents. Ann Cardiothorac Surg 2018;7(2): 273-83.

5) Weber AL, Rahemtullah A, Ferry JA. Hodgkin and non-Hodgkin lymphoma of the head and neck: Clinical, pathologic, and imaging evaluation. Neuroimaging Clin N Am 2003;13(3):371-92. 
6) Fallah M, Liu X, Ji J, Försti A, Sundquist K, Hemminki K. Autoimmune diseases associated with non-Hodgkin lymphoma: A nationwide cohort study. Ann Oncol 2014;25(10):2025-30.

7) Holm LE, Blomgren H, Löwhagen T. Cancer risks in patients with chronic lymphocytic thyroiditis. N Engl J Med 1985;312(10):601-4.

8) Varadharajan K, Mathew R, Odutoye B, Williamson P, Madden B. Use of tracheal stenting in the palliation of anaplastic thyroid carcinoma: Tertiary centre experience. J Laryngol Otol 2015; 129(6):568-71.

9) Hwang YC, Kim TY, Kim WB, Shong YK, Yi KH, Shong M, et al. Clinical characteristics of primary thyroid lymphoma in Koreans. Endocr J 2009;56(3):399-405.

10) Coiffier B, Lepage E, Briere J, Herbrecht R, Tilly H, Bouabdallah $\mathrm{R}$, et al. CHOP chemotherapy plus rituximab compared with $\mathrm{CHOP}$ alone in elderly patients with diffuse large-B-cell lymphoma. N Engl J Med 2002;346(4):235-42.

11) Persky DO, Unger JM, Spier CM, Stea B, LeBlanc M, McCarty MJ, et al. Phase II study of rituximab plus three cycles of CHOP and involved-field radiotherapy for patients with limited-stage aggressive B-cell lymphoma: Southwest Oncology Group study 0014. J Clin Oncol 2008;26(14):2258-63.

12) Shin JH, Baek JH, Oh YM, Ha EJ, Lee JH. Combination therapy of temporary tracheal stenting and radiofrequency ablation for multinodular thyroid goiter with airway compression. Korean $\mathrm{J}$ Radiol 2013;14(5):805-9.

13) Ding J, Chen $Z$, Shi M. Tracheal stenting for primary tracheal mucosa-associated lymphoid tissue lymphoma. Eur J Med Res 2013;18:8.

14) Madan K, Shrestha P, Garg R, Hadda V, Mohan A, Guleria R. Bronchoscopic management of critical central airway obstruction by thyroid cancer: Combination airway stenting using tracheal and inverted-Y carinal self-expanding metallic stents. Lung India 2017:34(2):202-5

15) Cho SB, Cha SA, Choi JY, Lee JM, Kang HH, Moon HS, et al. Serious complications after self-expandable metallic stent insertion in a patient with malignant lymphoma. Tuberc Respir Dis (Seoul) 2015;78(1):31-5. 